\title{
Prospects of combining adoptive cell immunotherapy with bortezomib
}

\author{
"...bortezomib could serve as an effective combinatorial treatment \\ along with other approaches of cancer immunotherapy to break \\ tumor-induced tolerance in $\mathrm{CD}^{+} \mathrm{T}$ cells and elicit a robust antitumor \\ immune response in cancer patients."
}

First draft submitted: 20 January 2017; Accepted for publication: 24 January 2017; Published online: 17 March 2017

Keywords: adoptive T-cell therapy • cancer immunology • cellular immunology

Bortezomib, also known as PS-341 and Velcade $^{\mathrm{TM}}$, is a front-line drug approved in the USA and EU for the treatment of multiple myeloma [1] and mantle cell lymphoma [2]. Its use has been extended lately to advanced stage non-small-cell lung cancer [3]. Bortezomib inhibits the ubiquitin-proteasome proteolytic pathway, which cancer cells rely heavily upon due to their high rate of aberrant protein generation and need of rapid protein turnover. Therefore, interference with the proteasome system became an attractive therapeutic strategy against cancer. Intracellular pathways requiring protein degradation for activation, such as the $\mathrm{NF \kappa B}$ pathway, which maintain cancer cell resistance to apoptosis, are also one of the targets of this drug. The reversible nature of proteasome inhibition by bortezomib sets it apart from other commercially available proteasome inhibitor drugs. While bortezomib's boronic acid moiety prevents it from being transported out of the cell by the multidrug resistance system, its transient half-life (9-15 h) makes it tolerable to patients when given at the doses of $1 \mathrm{mg} / \mathrm{kg}$ body weight, approximately $15-20 \mathrm{nM}$ by blood volume. Bortezomib can sensitize solid tumor cells to apoptosis by amplifying tumor cell caspase- 8 activation in the deathinducing signaling complex following death receptor ligation by death ligands such as TRAIL or its receptor agonist monoclonal antibodies $[4,5]$. Unfortunately, clinical trials in patients with solid tumors showed that bortezomib lacks therapeutic efficacy as a single agent. Moreover, there were concerns over the negative side effects reported on immune cells, particularly on $\mathrm{CD}^{+}{ }^{+} \mathrm{T}$ cells and dendritic cells, when using high doses of bortezomib [6-8].

New data show that administration of the proteasome inhibitor bortezomib $(1 \mathrm{mg} / \mathrm{kg}$ body weight; $\leq 20 \mathrm{nM}$ ) has complementary immunostimulatory effects following adoptive transfer of $\mathrm{CD}^{+} \mathrm{T}$ cells in mice bearing kidney or mammary adenocarcinomas. It was observed that lymphocyte effector functions were significantly improved following bortezomib treatment by mechanisms involving the upregulation of multiple Notch components and lymphocyte activation signaling molecules $[9,10]$. These results are in contrast with the effects of bortezomib observed in vitro where the doses used were high $(>100 \mathrm{nM})$ - doses that cannot be extrapolated to the therapeutic situation in vivo. Only 15-20 nM concentrations of bortezomib are found to be tolerable in vivo in both mice and humans [5,11]. Growing evidence suggests that bortezomib given at 15-20 nM doses exhibits an intrinsic ability to increase the levels of immunostimulatory cytokines, and components of Notch and $\mathrm{NF} \kappa \mathrm{B}$ signaling pathways in lymphocytes, thereby amplifying their effector functions either directly or indirectly. Thus, bortezomib could be an attractive candidate for exploration in combination with antitumor T-cell and/or natural killer cell adoptive therapy $[12,13]$.
Samuel T Pellom Jr1,2,3, Ashutosh Singhal ${ }^{1}$ \& Anil Shanker ${ }^{*}, 1,3,4,5,6$

'Department of Biochemistry \& Cancer Biology, School of Medicine, Meharry Medical College, Nashville, TN, USA 2Department of Microbiology \& Immunology, School of Medicine, Meharry Medical College, Nashville, TN, USA

${ }^{3}$ School of Graduate Studies \& Research, Meharry Medical College, Nashville, TN, USA

${ }^{4}$ Host-Tumor Interactions Research Program, Vanderbilt-Ingram Comprehensive Cancer Center, Vanderbilt University, Nashville, TN, USA ${ }^{5}$ Vanderbilt Center for Immunobiology, Vanderbilt University, Nashville, TN, USA ${ }^{6}$ Vanderbilt Center for Translational \& Clinical Immunology, Vanderbilt University, Nashville, TN, USA *Author for correspondence: Tel.: +1 6153276460 Fax: +16153276442 ashanker@mmc.edu 
Augmentation of lymphocyte-stimulatory cytokine signaling in the tumor environment by bortezomib opens the possibility of overcoming tumor-induced suppression of numerous immune regulatory networks, which renders immunotherapy inefficient. Recently published studies evaluated therapeutic efficacy of the combination of bortezomib and adoptive T-cell transfer in mice with established renal or mammary adenocarcinoma tumors, which expressed defined low-avidity epitopes of model antigen hemagglutinin (HA) [14]. Notably, adoptive transfer of $\mathrm{CD}^{+}$ $\mathrm{T}$ or $\mathrm{CD}^{+} \mathrm{T}$ cells from MHC class I and II-restricted HA-specific T-cell receptor-transgenic Cln 4 and $6.5^{+/+}$ mice, respectively, followed by bortezomib administration resulted in reduced numbers of metastatic tumor pulmonary nodules and enhanced survival of tumorbearing mice. No negative effect on the number and viability of $\mathrm{CD}^{+}{ }^{+}$or $\mathrm{CD}^{+} \mathrm{T}$ lymphocytes, or tissueresident $\mathrm{CD} 11 \mathrm{c}^{+} \mathrm{CD} 8{ }^{+}$dendritic cells was observed in tumor-bearing mice. Nor did bortezomib affect the expression of costimulatory molecules on dendritic cells or MHC class I or II-associated antigen presentation to HA-specific T cells. In addition to direct antitumor effects, bortezomib had stimulatory effects on the host immune system. Data also suggest that bortezomib stabilized expression of IL2 receptor- $\alpha$ and intracellular T-cell receptor $\mathrm{CD} 3 \zeta$ chains, enhanced IFN- $\gamma$ production and sustained FasL-mediated CD8 ${ }^{+} \mathrm{T}$-cell cytotoxicity in tumor-bearing mice. These effects correlated with increased phosphorylation of nuclear and cytoplasmic NFкB p65 levels in CD8 ${ }^{+} \mathrm{T}$ cells, likely due to the inhibition of their proteasomal degradation by bortezomib. Moreover, a high surface expression of Fas was observed on tumor cells in bortezomib-administered mice, and in human melanoma tissue from a responsive patient who received combination therapy that included bortezomib. These findings underscore the high clinical potential of an optimized regime of bortezomib administration for enhancing $\mathrm{CD} 8^{+} \mathrm{T}$-cell function, thereby improving the outcome of adoptive T-cell therapy of cancer.

“

"...bortezomib can act as a safe and potent immunotherapeutic drug in sustaining antitumor immune effector functions and in addressing tumor-associated immunosuppression..."

Another important lymphocyte-stimulatory feature of bortezomib administration that was observed in these studies was the upregulation of the components of Notch signaling that plays a crucial role in functional differentiation and maturation of $\mathrm{T}$ cells among several other key biological processes [10]. Tumors downregulate Notch signaling in lymphocytes to suppress their effector function [15]. In various mouse tumor models, analysis of endogenous antitumor $\mathrm{CD} 8^{+}$ T-cell response after bortezomib treatment showed that bortezomib can rescue tumor-induced downregulation of Notch in lymphocytes while enhancing their effector function. $\mathrm{CD} 8^{+} \mathrm{T}$ cells in tumor-bearing mice following bortezomib administration not only showed increased Notch $1 / 2$ activation but also exhibited increased expression of T-cell activation molecules CD25 and CD 44, with significantly improved production of IFN- $\gamma$ and expression of eomesodermin, perforin and granzyme-B. T-box transcription factor eomesodermin is not only important for the differentiation of cytolytic $\mathrm{CD}^{+} \mathrm{T}$ cells, but also maintains the memory $\mathrm{CD}^{+}{ }^{+}$T-cell repertoire [16]. These bortezomibmediated enhancements of $\mathrm{CD}^{+}{ }^{+} \mathrm{T}$-cell effects associate with increased crosstalk between Notch and NFKB signaling pathways important for T-cell cytolytic function $[17,18]$. The findings predict that bortezomib can act as a safe and potent immunotherapeutic drug in sustaining antitumor immune effector functions and in addressing tumor-associated immunosuppression if well optimized in conjunction with other immune therapies.

Immunosuppressive chronic inflammation in the tumor microenvironment dampens immune effector response by interfering with immunostimulatory Notch and cytokine signaling. This diminishes the outcome of anticancer therapeutic protocols. It was observed that bortezomib affected cytokine production in the tumor microenvironment, thereby polarizing the cytokine milieu in favor of antitumor T-cell immunity to reduce tumor burden. Using a therapeutic regimen that mimics the clinic [5], bortezomib administration in tumor-bearing mice at a dose of $1 \mathrm{mg} / \mathrm{kg}$ body weight increased the levels of immunostimulatory cytokines IL-2, IL-12 and IL-15 and enhanced their downstream signaling by increasing the expression of their receptors on tumor-infiltrating $\mathrm{CD} 8^{+} \mathrm{T}$ cells in both endogenous or adoptive transfer of $\mathrm{CD}^{+} \mathrm{T}$ cells specific to a lowavidity $\mathrm{HA}_{518-526}$ epitope, resulting in reduced pulmonary metastatic nodules [19]. Specifically, bortezomib treatment increased IL-2 production by $\mathrm{CD} 4^{+} \mathrm{T}$ cells, IL-12 and IL-15 production from CD11c ${ }^{+}$dendritic cells, and enhanced the expression of IL-2 receptor- $\alpha$ chain, IFN- $\gamma$, granzyme-B, as well as the transcription factor eomesodermin on endogenous or adoptively transferred HA-specific CD8 ${ }^{+} \mathrm{T}$ cells from bortezomib-treated tumor-bearing mice. These effects of bortezomib treatment were supported by the activation of downstream PI3K/Akt/STAT5 pathways observed in $\mathrm{CD}^{+} \mathrm{T}$ cells following bortezomib treatment. Interestingly, bortezomib treatment restored IFN- $\gamma$ synthesis and inhibited metastasis in tumor-bearing mice 
even in the presence of neutralizing antibodies to IL-12 and IL-15. This indicates that bortezomib can activate alternative effector pathways that could strengthen tumor immune surveillance. These observations highlight the immunostimulatory potential of bortezomib with a capacity to sustain $\mathrm{CD}^{+}{ }^{+} \mathrm{T}$-cell effector function in the tumor microenvironment of solid tumors independent of the type of tumor.

Therefore, besides the established role of bortezomib in sensitizing tumors to cell death, it may act as a multifaceted immunomodulatory drug and can provide immune stimulatory effects along with immunotherapeutic strategies such as adoptive T-cell therapy. Moreover, by suppressing constitutive activation of NFKB in tumor cells, it also abrogates their resistance to cell death [20]. By its direct antitumor activity and intrinsic nature to modulate immune cells, bortezomib acts to release brakes on the immune system from the tumorassociated suppressive microenvironment, thereby making the combination therapy including bortezomib potentially more efficient than either therapy alone. Therapeutic restoration of lymphocytic Notch signaling and effector function could enhance antitumor

\section{References}

1 Bross PF, Kane R, Farrell AT et al. Approval summary for bortezomib for injection in the treatment of multiple myeloma. Clin. Cancer Res. 10(12 Pt 1), 3954-3964 (2004).

2 Kane RC, Dagher R, Farrell A et al. Bortezomib for the treatment of mantle cell lymphoma. Clin. Cancer Res. 13(18 Pt 1), 5291-5294 (2007).

3 Li T, Ho L, Piperdi B et al. Phase II study of the proteasome inhibitor bortezomib (PS-341, Velcade) in chemotherapynaive patients with advanced stage non-small cell lung cancer (NSCLC). Lung Cancer 68(1), 89-93 (2010).

4 Brooks AD, Jacobsen KM, Li W, Shanker A, Sayers TJ. Bortezomib sensitizes human renal cell carcinomas to TRAIL apoptosis through increased activation of caspase- 8 in the death-inducing signaling complex. Mol. Cancer Res. 8(5), 729-738 (2010).

5 Shanker A, Brooks AD, Tristan CA et al. Treating metastatic solid tumors with bortezomib and a tumor necrosis factorrelated apoptosis-inducing ligand receptor agonist antibody. J. Natl Cancer Inst. 100(9), 649-662 (2008).

6 Berges C, Haberstock H, Fuchs D et al. Proteasome inhibition suppresses essential immune functions of human CD4 + T cells. Immunology 124(2), 234-246 (2008).

7 Nencioni A, Garuti A, Schwarzenberg K et al. Proteasome inhibitor-induced apoptosis in human monocyte-derived dendritic cells. Eur. J. Immunol. 36(3), 681-689 (2006).

8 Straube C, Wehner R, Wendisch M et al. Bortezomib significantly impairs the immunostimulatory capacity of human myeloid blood dendritic cells. Leukemia 21(7), 1464-1471 (2007). immune surveillance following a carefully optimized bortezomib regimen, which could abrogate tumor resistance to cytolysis and overcome tumor-associated immunosuppression. Thus, by restoring the Notch$\mathrm{NF \kappa B}$ mediated effector signaling and increasing the immunostimulatory signaling via cytokines such as IL-2, IL-12 and IL-15 in antitumor CD8 ${ }^{+} \mathrm{T}$ cells, bortezomib could serve as an effective combinatorial treatment along with other approaches of cancer immunotherapy to break tumor-induced tolerance in $\mathrm{CD} 8^{+} \mathrm{T}$ cells and elicit a robust antitumor immune response in cancer patients.

\section{Financial \& competing interests disclosure}

This work was supported by funds to A Shanker by the following NIH grants U54 CA163069, U54 MD007593, SC1 CA182843 and R01 CA175370. The authors have no other relevant affiliations or financial involvement with any organization or entity with a financial interest in or financial conflict with the subject matter or materials discussed in the manuscript apart from those disclosed.

No writing assistance was utilized in the production of this manuscript.

Shanker A, Pellom ST Jr, Dudimah DF et al. Bortezomib improves adoptive T-cell therapy by sensitizing cancer cells to FasL cytotoxicity. Cancer Res. 75(24), 5260-5272 (2015).

10 Thounaojam MC, Dudimah DF, Pellom ST Jr et al. Bortezomib enhances expression of effector molecules in anti-tumor CD8 + T lymphocytes by promoting Notchnuclear factor-kappaB crosstalk. Oncotarget 6(32), 3243932455 (2015).

11 Hideshima T, Richardson P, Chauhan D et al. The proteasome inhibitor PS-341 inhibits growth, induces apoptosis, and overcomes drug resistance in human multiple myeloma cells. Cancer Res. 61(7), 3071-3076 (2001).

12 Ames E, Hallett WH, Murphy WJ. Sensitization of human breast cancer cells to natural killer cell-mediated cytotoxicity by proteasome inhibition. Clin. Exp. Immunol. 155(3), 504-513 (2009).

13 Seeger JM, Schmidt P, Brinkmann K et al. The proteasome inhibitor bortezomib sensitizes melanoma cells toward adoptive CTL attack. Cancer Res. 70 (5), 1825-1834 (2010).

14 Morgan DJ, Kreuwel HT, Fleck S, Levitsky HI, Pardoll DM, Sherman LA. Activation of low avidity CTL specific for a self epitope results in tumor rejection but not autoimmunity. $J$. Immunol. 160(2), 643-651 (1998).

15 Huang Y, Lin L, Shanker A et al. Resuscitating cancer immunosurveillance: selective stimulation of DLL1Notch signaling in T cells rescues T-cell function and inhibits tumor growth. Cancer Res. 71(19), 6122-6131 (2011). 
16 Buggert M, Tauriainen J, Yamamoto T et al. T-bet and Eomes are differentially linked to the exhausted phenotype of CD8 + T cells in HIV infection. PLoS Pathog. 10(7), e1004251 (2014).

17 Deftos ML, Bevan MJ. Notch signaling in T cell development. Curr. Opin. Immunol. 12(2), 166-172 (2000)

18 Hettmann T, Leiden JM. NF-kappa B is required for the positive selection of $\mathrm{CD}^{+}$thymocytes. J. Immunol. 165(9), 5004-5010 (2000).
19 Pellom ST, Dudimah DF, Thounaojam MC et al. Bortezomib augments lymphocyte stimulatory cytokine signaling in the tumor microenvironment to sustain CD8+T cell antitumor function. Oncotarget doi:10.18632/ oncotarget.14365 (2016) (Epub ahead of print).

20 Richardson PG, Barlogie B, Berenson J et al. A Phase 2 study of bortezomib in relapsed, refractory myeloma. N. Engl. J. Med. 348(26), 2609-2617 (2003). 